\title{
Properties of polypyrrole polyvinilsulfate films for dual actuator sensing systems
}

\author{
Victor H. Pascual*a ${ }^{*}$, Toribio F. Otero ${ }^{a}$, J. Schumacher ${ }^{\mathrm{b}}$ \\ a Laboratory of Electrochemistry and Intelligent Materials, Technical University of Cartagena, ETSII, \\ Campus Alfonso XIII, 30203, Cartagena, Spain.; ' Arquimea Ingeniería, Margarita Salas 10, 28919 , \\ Leganés, Spain.
}

\begin{abstract}
One of the challenges of modern science is the development of actuators able to sense working conditions while actuation, mimicking the way in which biological organs work. Actuation of those organs includes nervous (electric) pulses dense reactive gels, chemical reactions exchange of ions and solvent. For that purpose, conducting polymers are being widely studied. In this work the properties of self-supported films of the polypyrrole:polyvinilsulfate (PPy/PVS) blend polymer were assessed. X-ray photoelectron spectroscopy (XPS) studies show how during reduction / oxidation the polymer exchanges cations when immersed in a $\mathrm{NaClO}_{4}$ aqueous solution, revealing free positive charges in the electrolytic solution as the driving agents leading to the swelling/shrinking of the polymer. Eventually it is the phenomenon responsible of the actuation of the polymeric motors. Submitting the system to consecutive potential sweeps shows the reaction is really sensing the scan rate used in each cycle revealing that while actuating the system is actually sensing the electrochemical working conditions.
\end{abstract}

Keywords: conducting polymers, electro-chemo-biomimesis, artificial muscles, polypirrole

\section{INTRODUCTION}

An uninterrupted feedback communication about the working conditions between some organs and the brain is what originates proprioception $^{1,2}$. Living cells possess reactant macromolecules that through reactions (molecular motors) develop conformational movements ${ }^{3}$.

The last decades have witnessed the development of a plethora of electrochemical devices such as conducting polymers or graphenes ${ }^{4-6}$. When these reactions take place the relative proportions of ions, solvent and polymer changes leading to conformational movements, as it happens inside the biological cells ${ }^{7,8}$. These composition dependent properties are allowing the creation of new devices such as artificial muscles ${ }^{9,10}$, smart windows ${ }^{11,12}$ or biosensors ${ }^{13}$. Sensing the working conditions during actuation these artificial systems mimic biological ones ${ }^{14}$. In a physically uniform device several sensors and an actuator work simultaneously. The source of this dual behavior is the electrochemical reaction.

This work studies the electrogeneration and electrochemical properties of polypyrrole/polyvinylsulfate (PPy/PVS) films after having been removed from the metal electrode over which they were generated, as well as their sensing properties when submitted to potential sweep rates the scan rate varies.

\section{METHODOLOGY AND RESULTS}

\subsection{Chemicals and Equipment}

To electrogenerate the polymeric blend, the following substances were employed: distilled pyrrole (Fluka), Poly(vinylsulfonic acid, sodium salt) solution (NaPVS) (Sigma-Aldrich, $25 \mathrm{wt} \%$ in H2O), lithium and sodium perchlorate (Sigma-Aldrich) and Milli-Q water.

An Autolab potentiostat/galvanostat PGSTAT302 (NOVA 1.11 software) was employed. A one-compartment electrochemical cell with an $\mathrm{Ag} / \mathrm{AgCl}(3 \mathrm{M} \mathrm{KCl})$ reference electrode (Metrohm), two stainless steel AISI 316 with a total immersed area of $14.56 \mathrm{~cm}^{2}$ as counter electrodes surrounding a working electrode of the same material with a total immerse surface of $7.28 \mathrm{~cm}^{2}$

Electroactive Polymer Actuators and Devices (EAPAD) 2017, edited by Yoseph Bar-Cohen, Proc. of SPIE Vol. 10163, 101630D · C 2017 SPIE · CCC code: 0277-786X/17/\$18 · doi: 10.1117/12.2259913 
A Sartorious Extended ED224s balance was used to weigh the films and an electronic microscope Hitachi S.3500N was employed for X-ray photoelectron spectroscopy (XPS).

\subsection{Film Preparation}

$\mathrm{PPy} / \mathrm{PVS}$ films were generated at room temperature over the working electrode from a $0.05 \mathrm{M} \mathrm{NaPVS}$ and $0.1 \mathrm{M}$ pyrrole aqueous solution. But first a study was carried out to find suitable electrochemical conditions to synthesize the material.

The potential window of the electrolyte $(0.05 \mathrm{M} \mathrm{NaPVS}$ aqueous solution) was found by subjecting the solution to potential sweeps at $40 \mathrm{mVs}^{-1}$ in the potential interval $(-1200 \mathrm{mV}, 1000 \mathrm{mV})$. Figure 1 shows how if the interval is wider than $(-1100 \mathrm{mV}, 900 \mathrm{mV})$ the water electrolysis begins, either with hydrogen release if the cathodic limit is open or with oxygen release on the anodic side.

If the cycling is carried out with the $0.05 \mathrm{M} \mathrm{NaPVS}$ and $0.1 \mathrm{M}$ pyrrole aqueous solution the voltammetric responses reveal that the monomeric oxidation polymerization takes place for potentials over $650 \mathrm{mV}$ with a clean working electrode and over $400 \mathrm{mV}$ with a partially coated with monomer electrode. In Figure 1 it can also be seen that the first cycle exhibits a loop, indicating that the process start by nucleation. The oxidation-polymerization is produced between $400 \mathrm{mV}$ and 900 $\mathrm{mV}$, far enough from the limits where the water electrolysis started.

With this information, to electrogenerate the polymer blend, a potential of $800 \mathrm{mV}$ was first applied until a uniform film coated the metal and a charge of $0.5 \mathrm{C}$ was consumed. After that, the potential was lower to $500 \mathrm{mV}$ and kept for $37000 \mathrm{~s}$. This time was considered enough for the films to be removed from the metal. The mass of the rinsed and dry film generated this way was of $12.0 \mathrm{mg}$

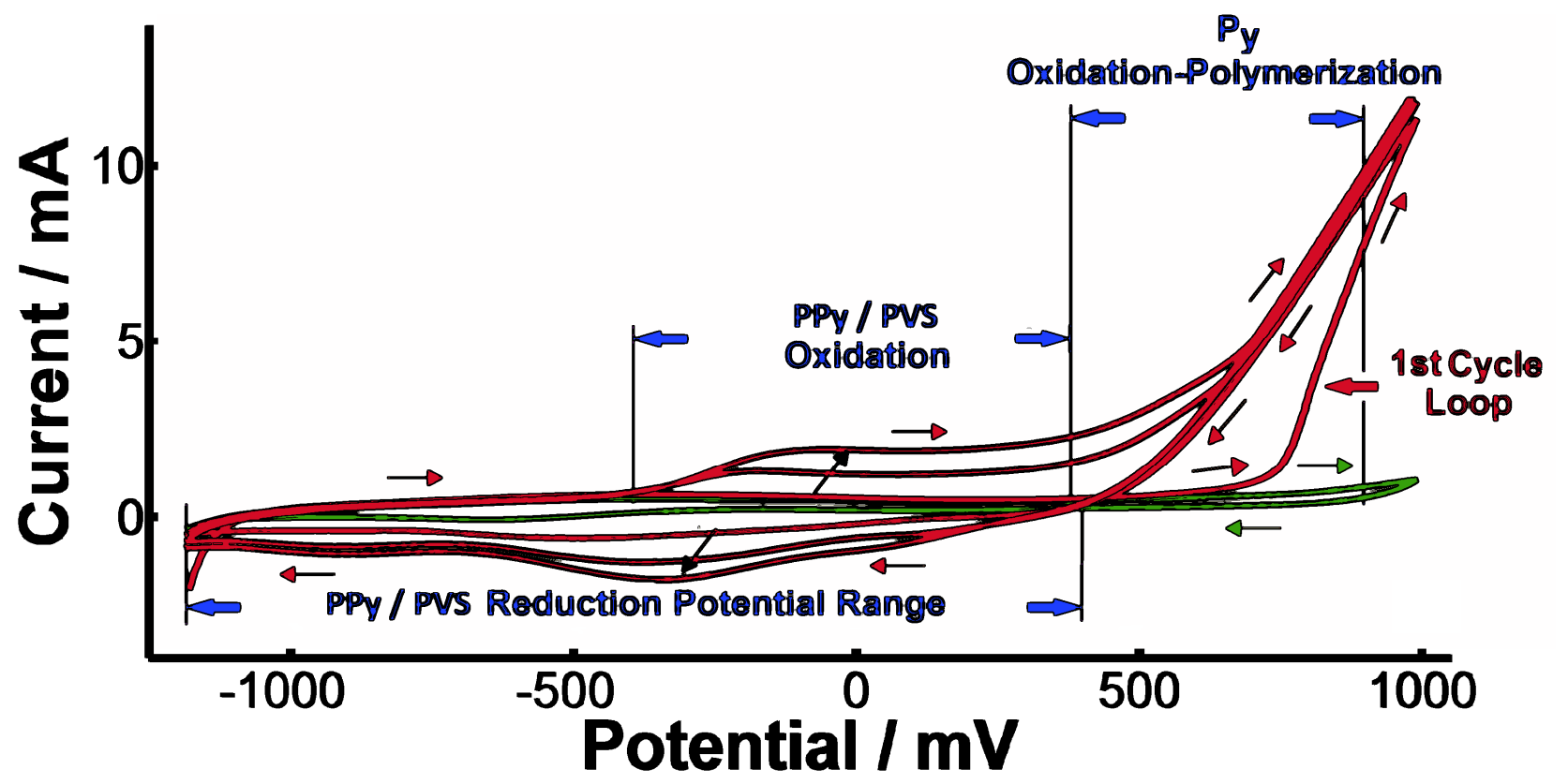

Figure 1. Voltammetric responses of the background electrolyte (green) (0.05 M NaPVS aqueous solution and the three first consecutive cycles in a $0.05 \mathrm{M}$ NaPVS and $0.1 \mathrm{M}$ pyrrole aqueous solution (red), at a scan rate of 40 $\mathrm{mVs}^{-1}$.

\subsection{Influence of the Cathodic Limit}

The Figure 2 shows the results of subjecting the film to a potential sweeps rates between a fixed anodic potential of 700 $\mathrm{mV}$ and a cathodic limit varying from $-600 \mathrm{mV}$ to $-1800 \mathrm{mV}$ in a $0.1 \mathrm{M} \mathrm{LiClO}_{4}$ aqueous solution. Stationary voltammetric responses (reached after two cycles) display a shift of the anodic peak towards more positive potentials. 
This phenomenon can be explained by the appearance of structures like lamellas, vesicles and micelles ${ }^{15}$, more stable from an energetic point of view. Since the only source of energy available is the potential (the experiments were realized at constant temperature, electrolyte concentration an so on,) to reoxidize these deep reduced formations a higher potential is needed $^{16-18}$. Due to a complete oxidation of the film erases its memory the reduction peak appears always at the same potential.

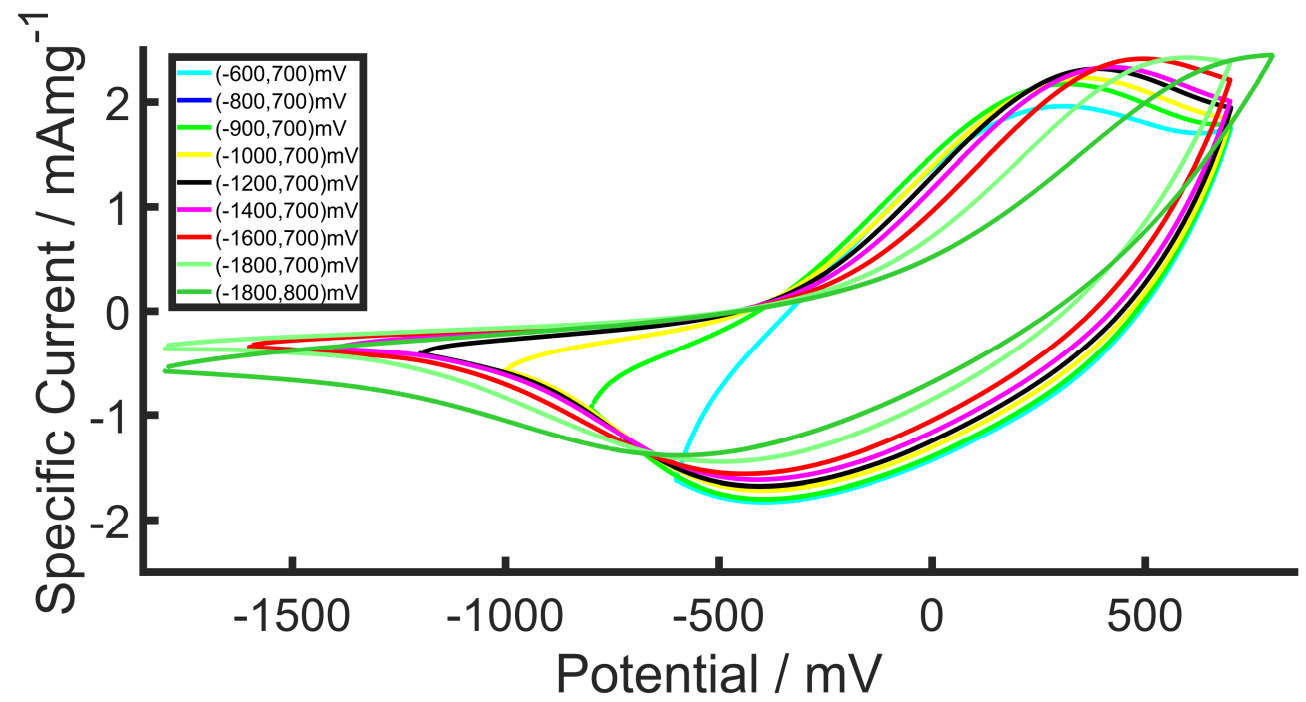

Figure 2. Voltammetric responses corresponding to a self-supported PPy/PVS immersed in a $0.1 \mathrm{M} \mathrm{LiClO}_{4}$ aqueous solution with a fixed anodic limit of the $700 \mathrm{mV}$ and a cathodic one going from $-600 \mathrm{mV}$ to $-1800 \mathrm{mV}$. Realized with a scan rate of $10 \mathrm{mVs}^{-1}$.

\subsection{Ionic Exchange}

To determine which kind of ions this polymeric blend exchanges when working immersed in a $0.1 \mathrm{M} \mathrm{NaClO}_{4}$ aqueous solution, three films were analyzed by XPS, in three different states, right after synthesis, after voltammetric reduction and after voltammetric oxidation. The results (Figure 3) shows a higher cation $\left(\mathrm{Na}^{+}\right)$content in the reduced sample indicating that the polymer is changing cations following the reaction

$$
\left[(P P y)_{S}\left(\mathrm{PVS}^{-} \mathrm{Na}^{+}\right)_{n}\left(\mathrm{H}_{2} \mathrm{O}\right)_{m}\right] \rightleftharpoons\left(\mathrm{PPy}^{n+}\right)\left(\mathrm{PVS}^{-}\right)_{n}+n N a^{+}+m\left(\mathrm{H}_{2} \mathrm{O}\right)+n e^{-}
$$

Being $P P y$ the active centers in the polymer that will accommodate a positive charge after oxidation, $P V S$ the anion that was trapped during the synthesis in the film and $\mathrm{Na}^{+}$the exchanged cation during the redox process. 


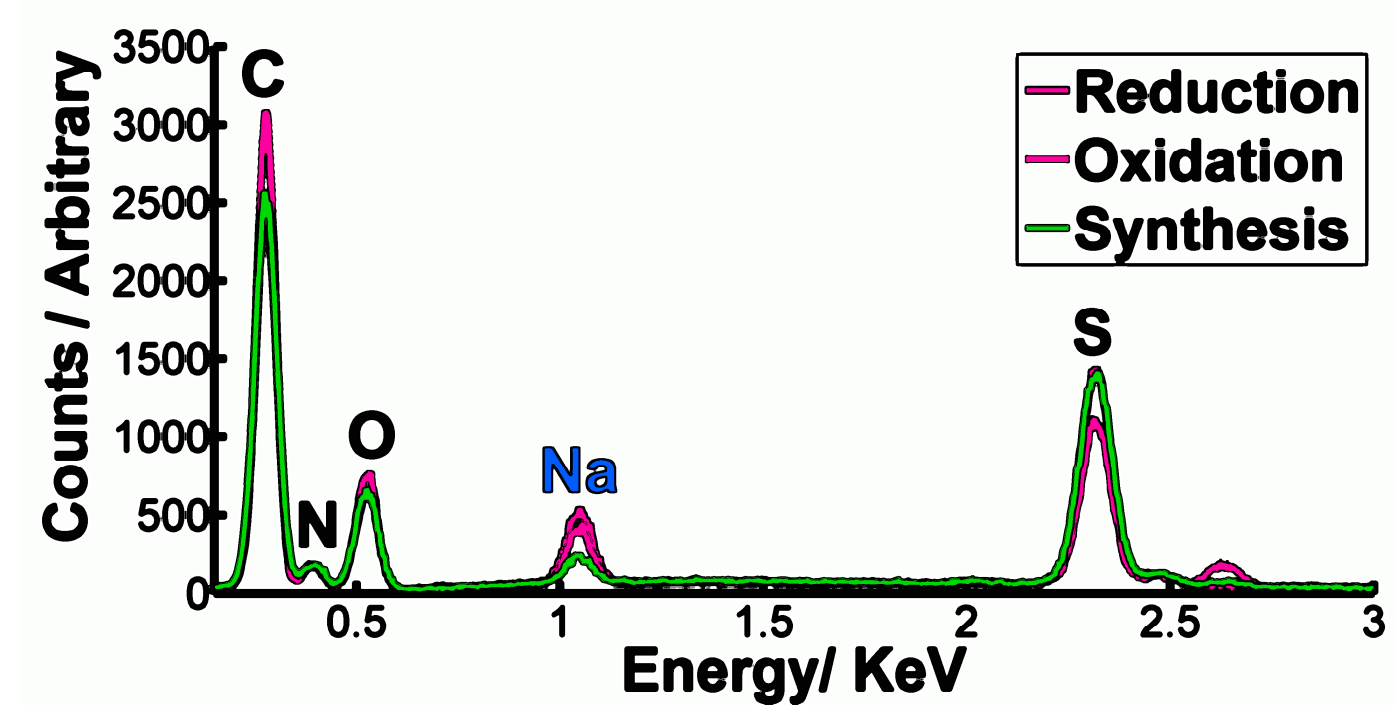

Figure 3. XPS analysis of three PPy/ PVS films in three different states, right after synthesis, oxidized and reduced.

\subsection{Influence of the Scan Rate}

After the study of some electrochemical properties of the PPy/PVS films, their sensing properties were assessed by changing the scan rate during cycling voltammetry experiences. Figure 4 a shows the stationary voltammetric (after two cycles) responses attained for scan rates ranging from $8 \mathrm{mVs}^{-1}$ to $100 \mathrm{mVs}^{-1}$ between the potential limits of $-800 \mathrm{mV}$ and $500 \mathrm{mV}$. In the graphic it can be seen clearly a scan rate $\left(20 \mathrm{mVs}^{-1}\right)$ above which the reduction and oxidation peaks have disappeared. Focusing on the lowest scan rates where these maxima are clearly depicted it can be appreciated how they separate from each other when the scan rate increases, shifting the oxidation peak to more anodic potentials and the cathodic peak to more cathodic. This phenomenon indicates the existence of a resistance to the entrance and exit of cations that raises with the scan rate.
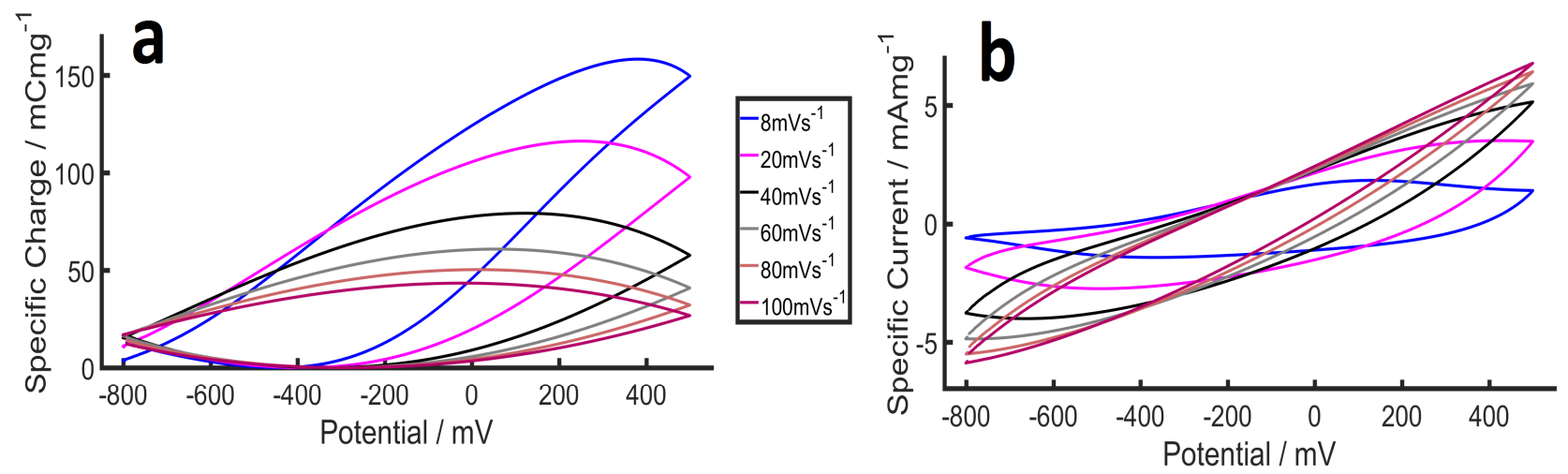

Figure 4. (a) Voltammetric responses for a self-supported PPy/PVS at different scan rates, from $8 \mathrm{mVs} \mathbf{s}^{-1}$ to $100 \mathrm{mVs}^{-1}$ in the potencial window $(-800,500) \mathrm{mV}$ (b) Concomitant coulovoltammetric responses.

Through integration of the voltammetric responses of Figure $4 \mathrm{a}$, the coulovoltammetric $(Q-E)$ are obtained. The close loop shown in the most part of these responses represent a region where the reduction positive charge equals the oxidize negative charge. The difference between the maximum and the minimum point of this region represents the reversible charge implicated in the oxidation/reduction of the polymer blend. The left open part represent the irreversible charge associated to other reactions like hydrogen evolution ${ }^{15}$. 
Figure 5 shows that, in fact, both charges decreases with the scan rate. Since other parameters such as as temperature or electrolyte concentration were kept constant during the experiment this reduction in the charge values with the scan rates indicate the presence of less electrochemical energy available per potential sweep for all reactions to take place.

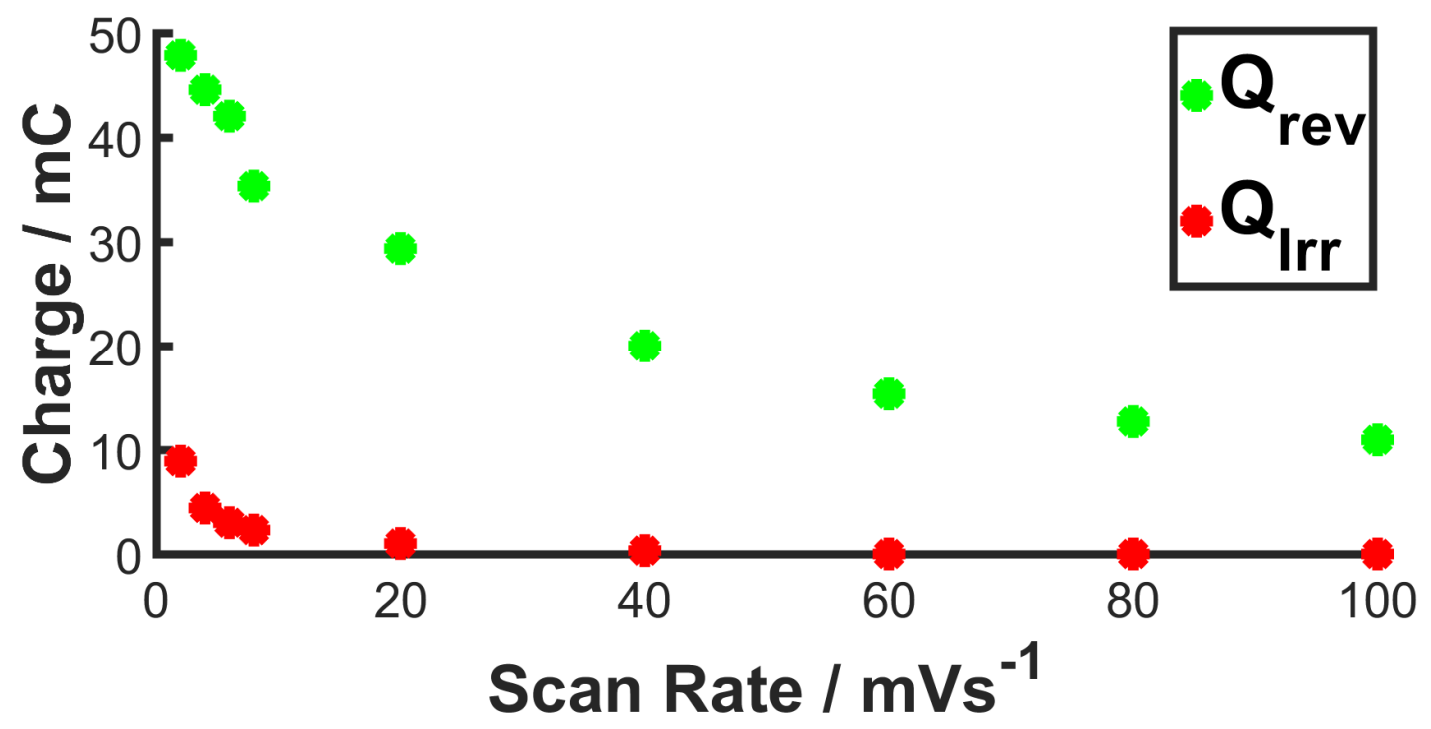

Figure 5. Evolution of the charge (reversible and irreversible with the scan rate

As mentioned, a part from the scan rate the other parameters (pressure, temperature,...) remain constant. In these conditions the effective electrochemical energy $(\Delta u)$ at which the film is subject can be expressed as

$\Delta u=\frac{\Delta U(J)}{v\left(V S^{-1}\right)}=\Delta Q(C) \frac{\Delta E(V)}{v\left(V s^{-1}\right)}=\frac{\Delta Q \cdot \Delta E}{v}(C s)$

Where $\mathrm{v}$ is the scan range, $\mathrm{E}$ the potential window and $\mathrm{Q}$, the reversible consumed charge, that according to the electrochemically stimulated conformational relaxation (ESCR) model is related to $\mathrm{v}$ through an exponential relation. The experimental results follow the equation

$$
\Delta u=a e^{b v}
$$

Where $a=11.14 \mathrm{~mJ} \mathrm{mV}^{-} \mathrm{s}, b=-0.084 \mathrm{mC}^{-1}$, and the correlation coefficient is $r^{2}=0.99$ (Figure 6) 


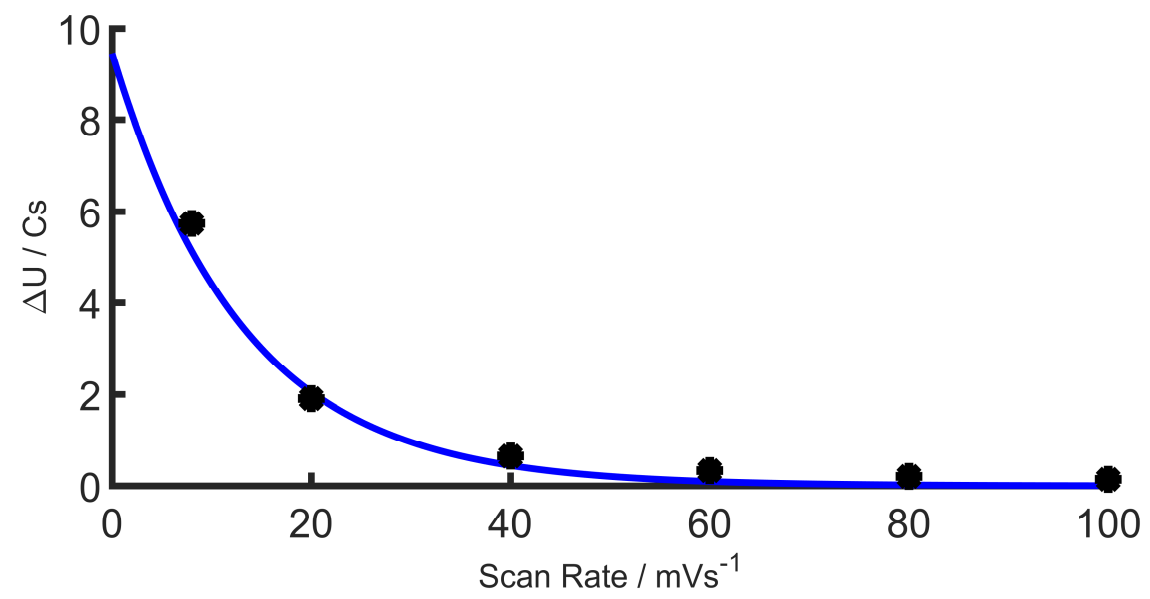

Figure 6. Evolution of the effective consumed electrical energy

The ESCR model establishes that there should be two exponential functions, one related to the conformational movements of the polymeric chains and other with the ion diffusion. What it happens in this case is that the scan rate range chosen is too high for the conformational oxidation/compaction and reduction/relaxation have enough time to occur. Under the conditions used the reaction controlled by diffusion.

The important consequence of this is that the electrical energy is actually sensing the working conditions, the scan rate in this case

\section{CONCLUSIONS}

Polymeric films of PPy/PVS thick enough to be peeled from the electrode were successfully generated and electrochemically studied showing indications of structures like lamelles or vesicles when the cathodic limit increases and the film is more and more reduced. This originates an anodic shift of the oxidation peak due to the need of reaching higher potentials to get the necessary energy to reoxidize. XPS analysis shows a cation exchange with the electrolyte. It was also proved that the electrochemical reaction is able to sense, through the electrical energy, the working conditions

\section{ACKNOWLEDGEMENT}

The research was supported by European Union's Horizon 2020 research and innovation program under the Marie Sklodowska-Curie grant agreement No 641822 .

\section{REFERENCES}

[1] Ashton-Miller, J. A., Wojtys, E. M., Huston, L. J.., Fry-Welch, D., “Can proprioception really be improved by exercises?,” Knee Surg. Sports Traumatol. Arthrosc. 9(3), 128-136 (2001).

[2] Lackner, J. R.., DiZio, P., "Vestibular, proprioceptive, and haptic contributions to spatial orientation," [Annual Review of Psychology], Annual Reviews, Palo Alto, 115-147 (2005).

[3] Barbara, J.-G.., Clarac, F., "Historical concepts on the relations between nerves and muscles," Brain Res. 1409, 3-22 (2011).

[4] Otero, T. F., "Conducting Polymers. Bioinspired inteligente materiales and devices" (2015).

[5] Otero, T. F., "Biomimetic Conducting Polymers: Synthesis, Materials, Properties, Functions, and Devices," Polym. Rev. 53(3), 311-351 (2013).

[6] Bar-Cohen, Y., Electroactive Polymer (EAP) Actuators as Artificial Muscles: Reality, Potential, and Challenges, SPIE Press (2001). 
[7] Otero, T. F.., Martinez, J. G., "Biomimetic intracellular matrix (ICM) materials, properties and functions. Full integration of actuators and sensors," J. Mater. Chem. B 1(1), 26-38 (2013).

[8] Otero, T. F., Martinez, J. G.., Arias-Pardilla, J., "Biomimetic electrochemistry from conducting polymers. A review Artificial muscles, smart membranes, smart drug delivery and computer/neuron interfaces," Electrochimica Acta 84, 112-128 (2012).

[9] Otero, T., Angulo, E., Rodriguez, J.., Santamaria, C., "Electrochemomechanical Properties from a Bilayer - Polypyrrole Nonconducting and Flexible Material Artificial Muscle," J. Electroanal. Chem. 341(1-2), 369-375 (1992).

[10] Mirfakhrai, T., Madden, J. D. W.., Baughman, R. H., "Polymer artificial muscles," Mater. Today 10(4), 30-38 (2007).

[11] Thakur, V. K., Ding, G., Ma, J., Lee, P. S.., Lu, X., "Hybrid Materials and Polymer Electrolytes for Electrochromic Device Applications," Adv. Mater. 24(30), 4071-4096 (2012).

[12] Rosseinsky, D. R.., Mortimer, R. J., "Electrochromic systems and the prospects for devices," Adv. Mater. 13(11), 783-+ (2001).

[13] Dhand, C., Das, M., Datta, M.., Malhotra, B. D., "Recent advances in polyaniline based biosensors," Biosens. Bioelectron. 26(6), 2811-2821 (2011).

[14] Otero, T. F.., Martinez, J. G., "Physical and chemical awareness from sensing polymeric artificial muscles. Experiments and modeling," Prog. Polym. Sci. 44, 62-78 (2015).

[15] Otero, T. F., Martinez, J. G., Fuchiwaki, M.., Valero, L., "Structural Electrochemistry from Freestanding Polypyrrole Films: Full Hydrogen Inhibition from Aqueous Solutions," Adv.

Funct. Mater. 24(9), 1265-1274 (2014).

[16] Fuchiwaki, M.., Otero, T. F., "Polypyrrole-para-phenolsulfonic acid/tape artificial muscle as a tool to clarify biomimetic driven reactions and ionic exchanges," J. Mater. Chem. B 2(14), 1954-1965 (2014).

[17] Otero, T. F., Alfaro, M., Martinez, V., Perez, M. A.., Martinez, J. G., "Biomimetic Structural Electrochemistry from Conducting Polymers: Processes, Charges, and Energies.

Coulovoltammetric Results from Films on Metals Revisited," Adv. Funct. Mater. 23(31), 3929-3940 (2013).

[18] Otero, T. F., Grande, H.., Rodriguez, J., "Role of conformational relaxation on the voltammetric behavior of polypyrrole. Experiments and mathematical model," J. Phys. Chem. B 101(42), 8525-8533 (1997). 